\title{
Precision neutron-interferometric measurement of the coherent neutron-scattering length in silicon
}

\author{
A. Ioffe,${ }^{1,2, *}$ D. L. Jacobson, ${ }^{3}$ M. Arif,,${ }^{3}$ M. Vrana, ${ }^{4}$ S. A. Werner, ${ }^{5}$ P. Fischer, ${ }^{1}$ G. L. Greene, ${ }^{6}$ and F. Mezei ${ }^{1}$ \\ ${ }^{1}$ Berlin Neutron Scattering Center, Hahn-Meitner-Institut, Glienicker Strasse 100, 14109 Berlin, Germany \\ ${ }^{2}$ St. Petersburg Nuclear Physics Institute, Gatchina, Leningrad District 188350, Russia \\ ${ }^{3}$ National Institute of Standards and Technology, Gaithersburg, Maryland 20899 \\ ${ }^{4}$ Nuclear Physics Institute of CAS, 20568 Rez, Czech Republic \\ ${ }^{5}$ Department of Physics and Astronomy, University of Missouri-Columbia, Columbia, Missouri 65211 \\ ${ }^{6}$ Los Alamos National Laboratory, Los Alamos, New Mexico 87545
}

(Received 15 August 1997)

\begin{abstract}
The neutron-interferometry (NI) technique provides a precise and direct way to measure the bound, coherent scattering lengths $b$ of low-energy neutrons in solids, liquids, or gases. The potential accuracy of NI to measure $b$ has not been fully realized in past experiments, due to systematic sources of error. We have used a method which eliminates two of the main sources of error to measure the scattering length of silicon with a relative standard uncertainty of $0.005 \%$. The resulting value, $b=4.1507(2) \mathrm{fm}$, is in agreement with the current accepted value, but has an uncertainty five times smaller. [S1050-2947(98)04808-2]
\end{abstract}

PACS number(s): 03.75.Dg, 07.60.Ly, 61.12.-q

\section{INTRODUCTION}

The coherent scattering length $b$ is the most important parameter characterizing the scattering of low-energy neutrons by nuclei in matter. An accurate knowledge of $b$ for different isotopes is essential for the application of neutronscattering methods in condensed-matter physics [1] and is crucial for a number of questions in fundamental physics [2]. Numerous techniques have been developed to measure $b$ for various elements. Shull and Oberteuffer [3] succeeded in measuring $b$ in $\mathrm{Si}$ with a relative standard uncertainty of $0.03 \%$ by observing Pendellösung interference fringes in dynamical diffraction, thus providing the most accurate value of the scattering length to date. The availability of large, high-purity, perfect crystals of Si has motivated the use of this element as a gauge material in evaluating techniques developed to determine $b$. In this paper we report the results of employing a neutron-interferometry method [4] to measure the scattering length of $\mathrm{Si}$, with an uncertainty reduced by a factor of 5 over the Shull-Oberteuffer measurement. This more widely applicable technique for improved accuracy in measuring $b$ is highly desirable, particularly for the study of hydrogen in metals [5] and the electrical properties of the neutron [6].

Neutron interferometry is extremely flexible and has been used to measure $b$ for many elements and their isotopes [7] in different aggregate states [8-10]. Samples are generally flat slabs, so the phase shift of the neutron wave traversing a sample in the dispersive geometry [Fig. 1(a)] is given by

$$
\Delta \phi_{s}=-N b \lambda D_{\mathrm{eff}},
$$

where $N$ is the atom density in the sample, $\lambda$ is the incident neutron wavelength, and $D_{\text {eff }}$ is the effective thickness of the

\footnotetext{
*Present address: Department of Physics \& Astronomy, University of Missouri-Columbia, Columbia, MO 65211 and National Institute of Standards and Technology, Gaithersburg, MD 20899.
}

sample traversed by the neutron beam. In many cases this phase shift can be measured easily with a precision of $0.01 \%$ or better; however, the accurate determination of $b$ requires that $\lambda$ be known with an equivalent accuracy. Typically the nominal (mean) wavelength of the beam traversing the interferometer is determined within a relative uncertainty of $0.1 \%$, thus the overall uncertainty achieved with the NI technique has been limited to about $0.1 \%$.

To eliminate the wavelength dependence Rauch et al. [11] proposed that the phase shift measurement could be performed in the nondispersive geometry shown in Fig. 1(b), in which the sample surfaces are arranged to be parallel to the Bragg planes of the interferometer crystal. In this case the effective thickness is $D_{\text {eff }}=D / \sin \theta_{B}$, which when substituted into Eq. (1) along with Bragg's law $\left(\lambda=2 d \sin \theta_{B}, d\right.$ is the interferometer Bragg plane spacing, $\theta_{B}$ is the Bragg angle of the interferometer) gives the wavelength-independent expression for the nondispersive phase shift:

$$
\Delta \phi_{s}=-2 d N b D \text {. }
$$

However, this method has been shown to be extremely sensitive to misalignment [4]. For the sample placed in beam path II with a small horizontal misalignment angle, $\Delta \varepsilon=\varepsilon$ $-\varepsilon_{0}$, of the sample surfaces with respect to the Bragg planes, one can express the near nondispersive phase difference for waves traversing path II relative to path $\mathrm{I}$ in the interferometer as

$$
\begin{aligned}
\Delta \phi(\varepsilon, \gamma) \equiv & \phi_{\mathrm{II}}-\phi_{\mathrm{I}}=-2 N b D d \frac{\sin \left(\theta_{B}\right)}{\sin \left(\theta_{B}+\Delta \varepsilon\right) \cos (\Delta \gamma)} \\
\approx & -\frac{2 N b D d}{\cos (\Delta \gamma)}\left\{1-\Delta \varepsilon \cot \left(\theta_{B}\right)\right. \\
& \left.+\frac{(\Delta \varepsilon)^{2}}{2}\left[1+2 \cot ^{2}\left(\theta_{B}\right)\right]\right\}
\end{aligned}
$$

where $\Delta \gamma=\gamma-\gamma_{0}$ is the vertical misalignment tilt angle. In the case of perfect horizontal alignment, $\Delta \varepsilon=0$, the phase 

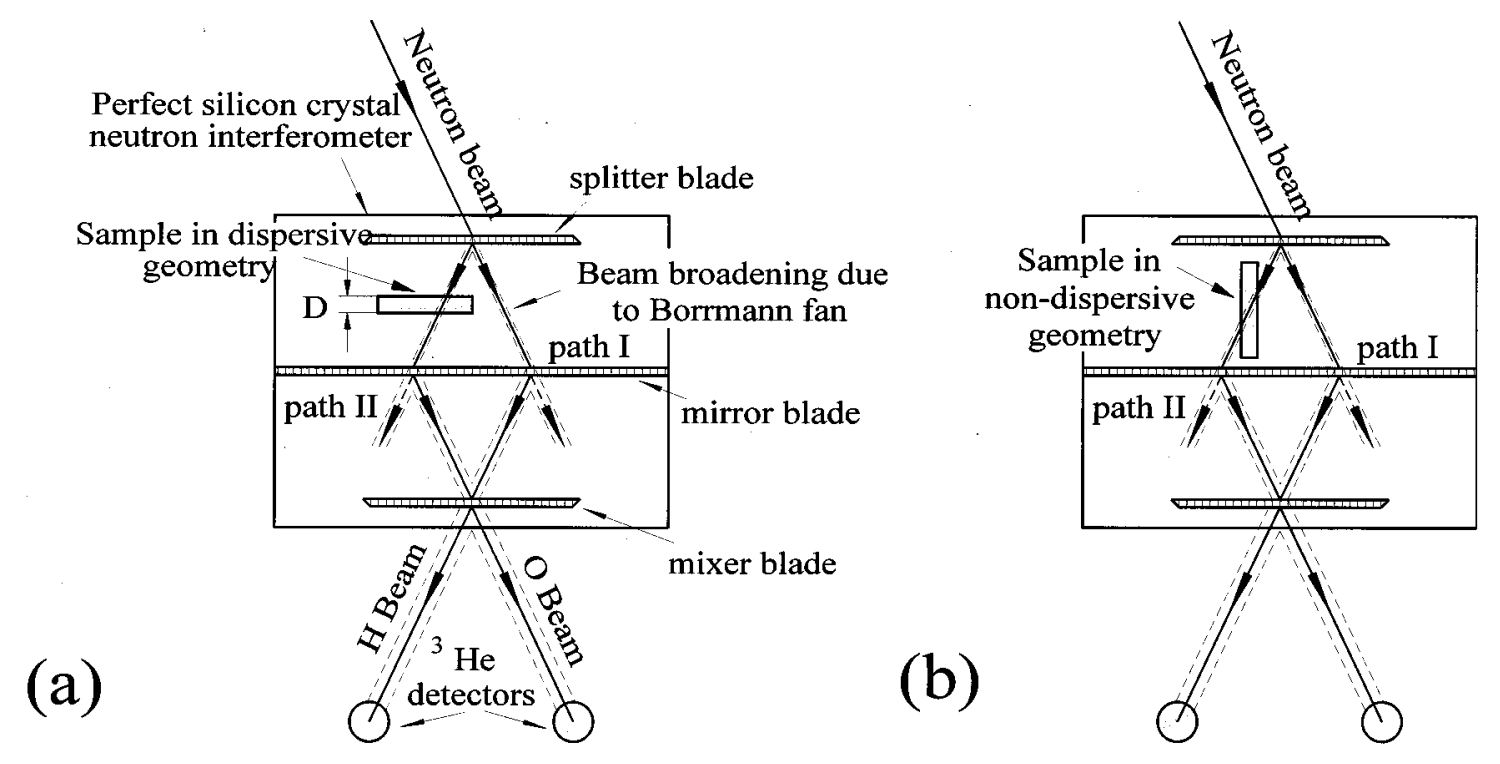

FIG. 1. Schematic view of (a) the dispersive phase shift geometry, and (b) the nondispersive, phase shift geometry.

shift $\Delta \phi=-2 N b D d / \cos (\Delta \gamma)$ is wavelength independent. In the case of misalignment, $\Delta \varepsilon \neq 0$, the second and third terms in Eq. (3) can be considered as small $\lambda$-dependent corrections. The accurate determination of $b$ in this case is mainly defined by terms linear in $\Delta \varepsilon$, so that $\Delta b / b=\Delta \varepsilon \cot \theta_{B}$. Therefore, in order to achieve a level of uncertainty of $0.005 \%$ or better in the measurement of $b$, the horizontal misalignment angle $\Delta \varepsilon$ must be less than $6 \operatorname{arc} \sec$ (for $\theta_{B}$ $=30^{\circ}$ ).

To overcome the alignment problem associated with the nondispersive geometry a method incorporating a LaueLaue-Laue (LLL) neutron interferometer has been proposed [4]. The principal idea of this method is to measure the nondispersive phase shift due to the sample, not relative to the empty path II as given by Eq. (3), but relative to the beam with the sample placed asymmetrically $(\Delta \varepsilon \rightarrow-\Delta \varepsilon)$. This is achieved by transporting the sample parallel to the surfaces of the interferometer blades to positions 1 and 2 shown in Fig. 2. In moving the sample from position 1 (beam path I) to position 2 (beam path II) the sign of $\Delta \varepsilon$ in Eq. (3) changes, and of course, there is an overall change of sign of $\Delta \phi$. Thus the difference of the phase shifts is

$$
\begin{aligned}
\Theta(\varepsilon, \gamma) & \equiv \Delta \phi_{1}-\Delta \phi_{2} \\
& \approx \frac{2 N b D d}{\cos (\Delta \gamma)}\left\{2+(\Delta \varepsilon)^{2}\left[1+2 \cot ^{2}\left(\theta_{B}\right)\right]\right\}
\end{aligned}
$$

which is a quadratic function of the small horizontal misalignment angle, $\Delta \varepsilon$. Using Eq. (4) has immediate advantages to Eq. (3). This difference $\Theta(\varepsilon, \gamma)$ of the phase shifts $\Delta \phi_{1}$ and $\Delta \phi_{2}$ is a function which is symmetric with respect to $\Delta \varepsilon$ and $\Delta \gamma$, allowing the determination of $\varepsilon_{0}$ and $\gamma_{0}$. The value of $b$ is obtained at the minimum of $\Theta(\varepsilon, \gamma)$ when $\Delta \varepsilon$ $=\Delta \gamma=0$, namely,

$$
b=\frac{\left(\Delta \phi_{1}-\Delta \phi_{2}\right)_{\min }}{4 N d D}=\frac{\Theta\left(\varepsilon_{0}, \gamma_{0}\right)}{4 N d D}
$$

It is important to note that the error in $b$, due to misalignment, is $\Delta b / b=\left[1+2 \cot ^{2}\left(\theta_{B}\right)\right](\Delta \varepsilon)^{2} / 2$, which is quadratic in $\Delta \varepsilon$. Therefore a misalignment of $\Delta \varepsilon \approx 0.2^{\circ}$ results in less than a $0.005 \%$ error in $b$ (for $\theta_{B}=30^{\circ}$ ). This method reduces or eliminates the errors due to dispersion and alignment in the phase shift measurement, so that the accuracy in the determination of $b$ depends mainly on one's knowledge of the sample atom density $N$ and thickness $D$.

\section{EXPERIMENT}

Experiments were performed at both the Neutron Interferometry and Optics Facility (NIOF) at the NIST Center for Neutron Research (NCNR) in Gaithersburg, MD and at the Neutron Interferometry Facility at the Berlin Neutron Scattering Center of the Hahn-Meitner-Institut (BENSC) in Berlin, Germany. Both facilities used the same single-crystal silicon sample and the same technique to perform the scattering length measurement. The NIST facility used an interferometer crystal operating with the (111) Bragg planes at a wavelength of $0.270 \mathrm{~nm}$. The BENSC facility used an interferometer crystal operating with the (220) Bragg planes at a wavelength of $0.198 \mathrm{~nm}$.

The experimental layout is shown in Fig. 2. The silicon sample was placed in the non-dispersive position inside the first half of the interferometer, where the beam broadening due to the Borrmann fans is smaller (see Fig. 1). To minimize any effects of thickness variations the region of the sample that the neutrons probed was defined by a $0.6-\mathrm{mm}-$ thick neutron-absorbing $\mathrm{Cd}$ mask, with a $6 \mathrm{~mm} \times 6 \mathrm{~mm}$ square opening, placed over the sample.

Initially the sample was aligned to be a few degrees out of the exact, nondispersive position. The experimental data were collected by rotating the phase flag (quartz at NIST and $\mathrm{Si}$ at HMI) to an angle $\delta$, and then driving the sample between the out position 0, and positions 1 and 2 (see Fig. 2). At each position of the sample the neutron intensities in the $\mathrm{O}$ and $\mathrm{H}$ beams were recorded. A set of three interferograms (intensity versus $\delta$ ) were simultaneously collected after hav- 


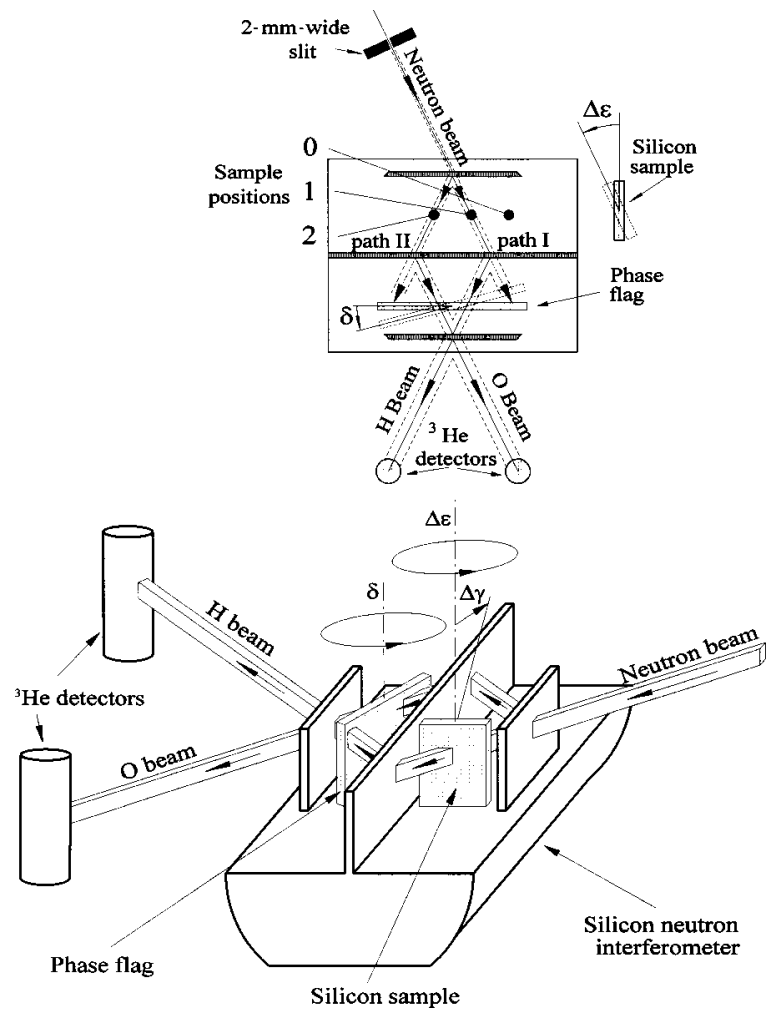

FIG. 2. Schematic and perspective views of the experimental setup. The three sample positions $(0,1$, and 2$)$ are shown.

ing rotated the phase flag through the stepping range (see Fig. 3); this data collection method minimizes the influence of phase drift of the interferometer. These interferograms were fitted to functions of the standard form

$$
I_{0}(\delta)=A\left\{1+C \cos \left[\Delta \Phi_{i}(\delta)\right]\right\},
$$

and

$$
I_{H}(\delta)=A\left\{1-C \cos \left[\Delta \Phi_{i}(\delta)\right]\right\}+B
$$

where

$$
\Delta \Phi_{i}(\delta)=f \delta+\Delta \phi_{i}+\Delta \beta_{0} .
$$

Here, the index $i(=0,1,2)$ gives the translation position of the sample, $f \delta$ is the phase shift due to the phase flag (appropriate for small $\delta$ ), $\Delta \phi_{i}$ is the phase shift due to the sample in position $i$, and $\Delta \beta_{0}$ is the empty interferometer phase difference. Thus for each O-beam interferogram there are four fitting parameters: the mean intensity $A$, the contrast $C$, the frequency $f$ of the phase flag interferogram, and the phase of the interferogram $\Delta \phi_{i}+\Delta \beta_{0}$. This sequence was repeated for various sample angles $\varepsilon$, holding the tilt $\gamma$ fixed, from which the quadratic dependence of $\Theta(\varepsilon)$ was obtained. The results are plotted in Fig. 4(a). From Fig. 4(a) the angle $\varepsilon_{0}$ for which the sample was nondispersively aligned was determined by a quadratic fit to $\Theta(\varepsilon)$. Once $\varepsilon_{0}$ was determined, the same alignment procedure was used to obtain the quadratic dependence of $\Theta\left(\varepsilon_{0}, \gamma\right)$ on $\gamma$, thus determining $\gamma_{0}$ [see Fig. 4(b)]. With the sample optimally aligned the phase shift in positions 1 and 2 was measured repeatedly. The resulting values of the difference phase are presented in Table I.
TABLE I. Results from the two experiments.

\begin{tabular}{lcc}
\hline \hline Facility & $\Theta\left(\varepsilon_{0}, \gamma_{0}\right)(\mathrm{rad})$ & $b(\mathrm{fm})$ \\
\hline HMI & $152 \pi-0.082(5)$ & $4.15102(21)$ \\
NIST & $248 \pi+0.455(7)$ & $4.15041(21)$ \\
\hline \hline
\end{tabular}

It should be pointed out that from a single interferogram it is not possible to determine how many multiples $n$ of $2 \pi$ that the phase of the neutron wave acquires from traversing the sample, so that

$$
\Delta \phi_{1,2}^{\prime}= \pm\left(\Delta \phi_{1,2}+2 \pi n\right)+\Delta \phi_{0} .
$$

In this case Eq. (5) should be rewritten as

$$
b=\frac{\left(\Delta \phi_{1}-\Delta \phi_{2}\right)_{\min }+4 \pi n}{4 N d D} .
$$

Thus depending on the choice of $n$ one can define a set of values for $b$, which satisfies Eq. (9). In order to determine the value for $n$ one should use low accuracy values of $b$ measured previously to within a relative standard uncertainty of better than about $0.5 \%$. The previous accepted result of 4.149(1) fm, for the scattering length $b$, in silicon, easily satisfied this requirement, and determined the value for $n$ $=62$ and 38 for NIST and HMI experiments, respectively, for the sample used in this experiment.

The uncertainty in $\Theta$ (see Table I) is due mainly to statistical variations and represents a relative standard uncertainty of about $0.0009 \%$. The systematic alignment error included in the combined relative uncertainty for $\Theta$ is much

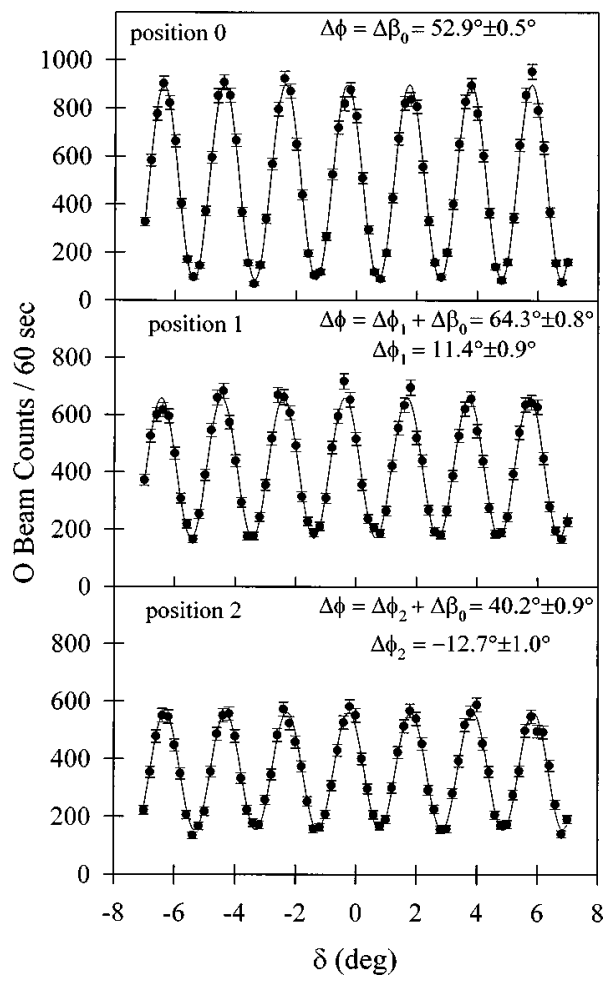

FIG. 3. Examples of interferograms taken at the three positions. The reduced contrast and intensity in positions 1 and 2 is due to the partial blocking of the neutron amplitude by the Cd mask placed on the sample. 

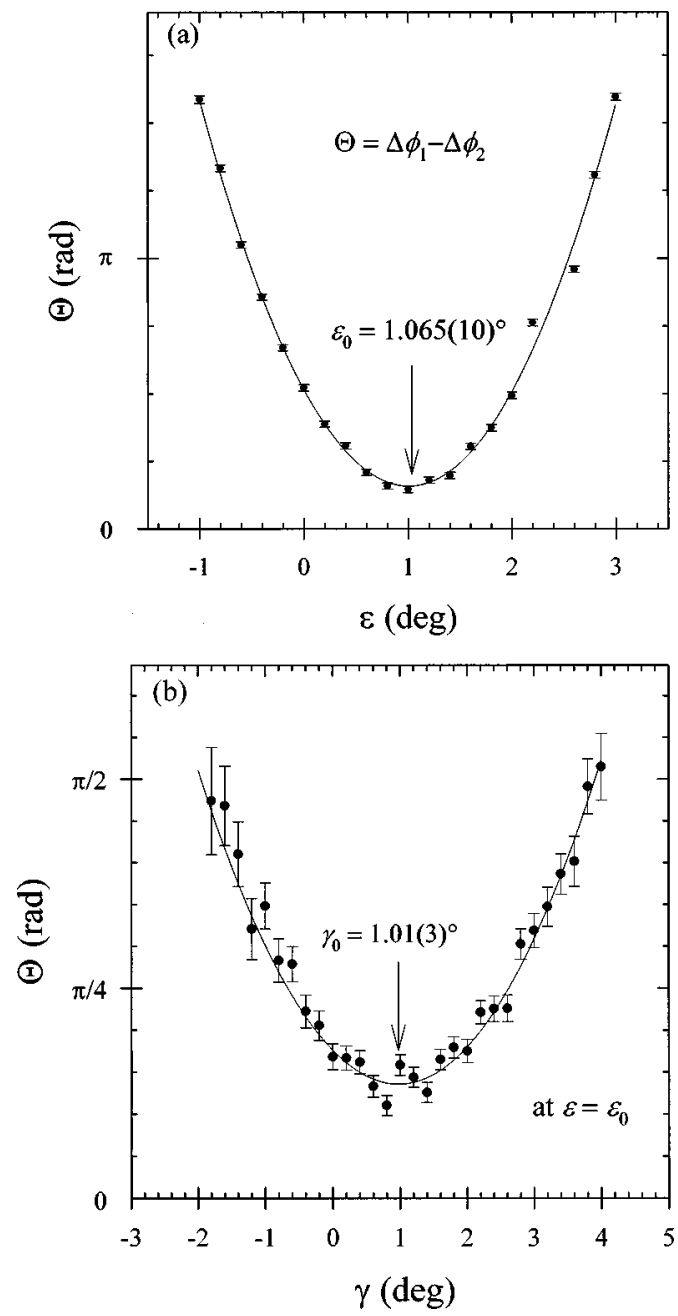

FIG. 4. The difference phase shift versus $\varepsilon$ (top) and $\gamma$ (bottom).

smaller and is roughly on the order of $0.0002 \%$. Since the experiment was performed in air and not in a vacuum an additional correction factor due to the displacement of air by the sample must be taken into account. The resulting formula for the scattering length is

$$
b=\frac{\Theta\left(\varepsilon_{0}, \gamma_{0}\right)}{4 N D d}+b_{\text {air }},
$$

where $b_{\text {air }} \equiv(N b)_{\text {air }} / N=9.137(9) \times 10^{-3}$ fm (calculated for the relative humidity of air of $20 \%[7,12]$ ) which is a significant correction at the level of experimental accuracy achieved in this experiment.
The values for the silicon atom density $N$ for experimental temperatures $26{ }^{\circ} \mathrm{C}$ and $19{ }^{\circ} \mathrm{C}$, respectively, have been adjusted from the crystal lattice spacing of silicon $a$ $=543101.993 \mathrm{fm}$ at $22.5^{\circ} \mathrm{C}$ by the thermal expansion coefficient of $2.567 \times 10^{-6} \mathrm{~K}^{-1}[13,14]$. The silicon sample thickness $D=0.30053(2) \mathrm{cm}$ was measured at the HMI. The variation in the sample thickness was determined by a high-resolution profile meter and was found to be less than $0.00001 \mathrm{~cm}$. A value for $D=0.300527(15) \mathrm{cm}$ was obtained in a separate measurement at the NIST Gauge Block Interferometer Facility in Gaithersburg, MD. From these measurements the most homogeneous area of the sample was selected to be used in the measurements. However, the systematic error due to variation in the sample thickness represents the main contribution to the combined relative uncertainty in the final result of about $0.005 \%$. Averaging the experimental values of $b$ obtained using two wavelengths leads to a final value for $b=4.1507(2) \mathrm{fm}$ in $\mathrm{Si}$, which is in reasonable agreement with the value 4.149 (1) fm obtained by Shull and Oberteuffer, but is five times more accurate.

\section{CONCLUSION}

The results of our experiments carried out using two different interferometers at different wavelengths by means of the new difference phase shift technique are in close agreement with each other, indicating no obvious systematic errors. In general the difference phase shift technique could be extended to greatly increase the accuracy of many previous, scattering length measurements which were limited by alignment errors and wavelength uncertainty errors. Because the systematic error due to the variation in the sample thickness represents the main contribution to the combined uncertainty in the final result, a more accurate value for $b$ of silicon could be obtained with a better sample.

\section{ACKNOWLEDGMENTS}

The authors would like to thank Dr. Theodore D. Doiron in the NIST Gauge Block Interferometer Facility for his expertise in measuring the silicon sample thickness. We would also like to thank Professor H. Rauch (Vienna) for providing the interferometer crystal for the HMI. A.I. and M.V. would like to acknowledge the support of BENSC and European Commission, TMR Programme, Network Contract No. ERBFMRXCT-0057 for "Neutron Optics." S.A.W. acknowledges support from NSF, Physics Division, Grant No. 9603559 .
[1] V. Sears, Neutron Optics (Oxford University Press, New York, 1989); G. E. Bacon, Neutron Diffraction (Clarendon, Oxford, 1979).

[2] L. Koester, in Neutron Physics, edited by G. Höhler and E. A. Niekisch, Springer Tracts in Modern Physics Vol. 80 (Springer-Verlag, Berlin, 1977), p. 1; S. A. Werner and A. G. Klein, in Methods of Experimental Physics, edited by K. Skold and D. Prince (Academic, Orlando, 1986), Vol. 23, Pt. A, p. 259.

[3] C. G. Shull and J. A. Oberteuffer, Phys. Rev. Lett. 29, 871 (1972); C. G. Shull, ibid. 21, 1585 (1968).
[4] A. Ioffe and M. Vrana, Phys. Lett. A 231, 319 (1997).

[5] H. Rauch, in Neutron Scattering from Hydrogen in Materials: Proceedings of the Second Summer School on Neutron Scattering, edited by Albert Furrer (World Scientific, Singapore, 1994), p. 31.

[6] A. Ioffe, M. Vrana, and V. Zabiyakin, J. Phys. Soc. Jpn. A65, 82 Suppl. (1996).

[7] L. Koester, H. Rauch, and E. Seymann, At. Data Nucl. Data Tables 49, 115 (1991).

[8] H. Kaiser, H. Rauch, G. Badurek, W. Bauspiess, and U. Bonse, Z. Phys. A 291, 231 (1979). 
[9] W. Bauspiess, U. Bonse, and H. Rauch, Nucl. Instrum. Methods 157, 495 (1978).

[10] H. Kaiser, M. Arif, and S. A. Werner, Physica B \& C 136B, 134 (1986).

[11] H. Rauch, E. Seidl, D. Tuppinger, D. Petrascheck, and R. Scherm, Z. Phys. B 69, 313 (1987).

[12] Handbook of Chemistry and Physics, edited by R. C. Weast,
M. J. Astle, and W. H. Bayer, 68th ed (Chemical Rubber, Boca Raton, FL, 1987), p. F-150.

[13] G. Basile, A. Bergamin, G. Cavagnero, G. Mana, E. Vittone, and G. Zosi, Phys. Rev. Lett. 72, 3133 (1994).

[14] P. Becker, K. Dorenwendt, G. Ebeling, R. Lauer, W. Lucas, R. Probst, H.-J. Rademacher, G. Reim, P. Seyfied, and H. Siegert, Phys. Rev. Lett. 46, 1540 (1981). 